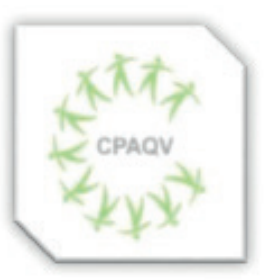

ISSN: 2178-7514

Vol. $13 \mid \mathbf{N}^{\circ} .2$ | Ano 2021
ARTIGO ORIGINAL

\section{ANÁLISE DO PERFIL CLÍNICO-EPIDEMIOLÓGICO DOS CASOS DE \\ HANSENÍASE NO BRASIL NO PERÍODO DE 2011-2020}

\section{Analysis of the clinical-epidemiological profile of leprosy cases in brazil in the 2011-2020}

Tereza Cristina dos Reis Ferreira ${ }^{1}$, Eduardo Augusto Silva Monteiro², Maria Fernanda Lourenço ${ }^{2}$, Rafael Lobato Machado $^{2}$, Thaisy Luane Gomes Pereira Braga ${ }^{2}$, Raquel de Souza Mota ${ }^{3}$, Paula Thayna Soares Lima ${ }^{3}$, Thatiane Belém Rosa ${ }^{3}$, Rafael da Rocha Dias Quintairos ${ }^{4}$, Aline Celloy Pará da Cruz ${ }^{4}$, Jeniffer de Alencar Pinheiro ${ }^{3}$, Shamyle Aramys dos Santos Costa ${ }^{5}$

\title{
RESUMO
}

A hanseníase permanece, ainda hoje, com consideráveis níveis de registros no Brasil, capaz de causar efeitos psicossociais permanentes e até mesmo incapacidades físicas. Objetivos: Analisar o perfil clínico-epidemiológico dos casos diagnosticados com Hanseníase no SINAN, relacionando-o com variáveis clínicas, geográficas e evolução da incidência da doença nos últimos 10 anos. Métodos: Trata-se de um estudo epidemiológico observacional e retrospectivo, em que os dados foram extraídos do SINAN. Resultados: Foram encontrados 867.687 casos de pacientes diagnosticados com hanseníase, de 2011 a 2020, com 2020 registrando cerca de 67\% de redução de casos em relação ao ano anterior. Dentre os pacientes, os maiores registros foram entre homens, pardos, de baixa escolaridade, entre 30 e 49 anos, da forma multibacilar e dimorfa, sendo que os maiores índices permaneceram nas Regiões Norte e Nordeste. Conclusão: A diminuição de registros do último ano pode ter sido por conta de maiores efetividades nos serviços de saúde ou por conta da pandemia de COVID-19, ainda assim, por conta da persistência do perfil epidemiológico relacionado ao baixo desenvolvimento social, evidencia-se a necessidade de melhorias da qualidade de vida da população com o objetivo de interromper o ciclo de transmissão da doença.

Palavras-chave: Hanseníase; Serviço de Saúde; Fatores Socioeconômicos; Atenção Primária à Saúde.

\begin{abstract}
Leprosy remains, even today, with considerable levels of records in Brazil, capable of causing permanent psychosocial effects and even physical disabilities. Objectives: To analyze the clinical-epidemiological profile of cases diagnosed with leprosy in the SINAN, relating it to clinical and geographic variables and evolution of the incidence of the disease in the last 10 years. Methods: This is an observational and retrospective epidemiological study, in which data were extracted from SINAN. Results: 867,687 cases of patients diagnosed with leprosy were found, from 2011 to 2020, with 2020 recording about $67 \%$ of cases reduction compared to the previous year. Among the patients, the highest records were among men, brown, with low education, between 30 and 49 years old, in the multibacillary and borderline form, with the highest rates remaining in the North and Northeast regions. Conclusion: The decrease in records in the last year may have been due to greater effectiveness in health services or due to the COVID-19 pandemic, yet, due to the persistence of the epidemiological profile related to low social development, it is evident the need to improve the population's quality of life in order to interrupt the disease transmission cycle.
\end{abstract}

Keywords: Leprosy; Health Service; Socioeconomic Factors; Primary Health Care

Autor de correspondência

1 Fisioterapeuta, Dra em Ciências da Reabilitação. Docente do curso de fisioterapia da Universidade do Estado do Pará- UEPA e da Faculdade Paraense de Ensino - FAPEN

2 Acadêmicos do Curso de Graduação em Medicina da Universidade do Estado do Pará- UEPA

3 Acadêmicos do Curso de Graduação de Fisioterapia da Universidade do Estado do Pará- UEPA.

4 Acadêmicos do Curso de Graduação de Fisioterapia da Faculdade Paraense de Ensino - FAPEN

5 Fisioterapeuta especialista em Terapia Intensiva, e docente do curso de Fisioterapia do Centro Universitário Leonardo da Vinci - Uniasselvi

Tereza Cristina dos Reis Ferreira

DOI: $10.36692 / \mathrm{v} 13 \mathrm{n} 2-23$ 


\section{INTRODUÇÃO}

A hanseníase é uma doença infectocontagiosa, crônica e milenar, a qual é causada pelo Mycobacterium leprae e, quando não diagnosticada ou tratada precocemente, é capaz de incapacitar fisicamente o indivíduo, assim como produzir uma série de sequelas psicossociais $^{1}$. De modo geral, o contágio decorre do contato prolongado entre um indivíduo bacilífero e outro suscetível por meio da via respiratória, atribuindo a essa doença alta infectividade, porém baixa patogenicidade2. Além disso, ao se analisar os fatores relacionados à infectividade da doença, percebe-se uma estreita relação entre os padrões socioeconômicos populacionais e a prevalência da hanseníase, o que demonstra importantes impactos decorrentes da pobreza, desigualdade e abandono pelos poderes públicos de certas parcelas populacionais ${ }^{2}$.

Nesse contexto, o impacto da pobreza, assim como o baixo acesso à informação e aos serviços públicos são, atualmente, os principais fatores relacionados à manutenção dessa doença no mundo ${ }^{3}$. Dentre esses aspectos, a inacessibilidade aos serviços de saúde, a qual gera um diagnóstico tardio e, consequentemente, agravamento do quadro clínico e das incapacitações provocadas pela hanseníase, como perda da acuidade visual, sensibilidade e força nos membros, além de cicatrizes, manchas e deformidades, representa um fator crucial para a compreensão da atual distribuição epidemiológica dessa enfermidade no país ${ }^{4}$. Ademais, a ausência do acompanhamento do indivíduo infectado leva ao maior contato deste com a comunidade, incluindo a sua própria família, o que é evidenciado na grande quantidade de contágios intrafamiliares ${ }^{2}$. Também contribui ao complexo contexto social associado à hanseníase, o seu alto poder incapacitante, abrangendo uma ampla parcela de indivíduos em idade economicamente ativa, cujo resultado é a retirada desse indivíduo do mercado de trabalho, diminuição do poder aquisitivo familiar e manutenção da pobreza, além de dispendiosos gastos governamentais na reabilitação e cuidado a esses pacientes ${ }^{5}$.

No mundo, a hanseníase permanece como uma doença endêmica, em que foram registrados, em 2018, 208.619 novos casos em 161 países, apresentando uma taxa de 2,74 casos/100 mil e prevalência de 0,29/10 mil, com a maioria desses casos localizados em países em desenvolvimento ou com amplos grupos populacionais sem acesso à saúde ou educação de qualidade ${ }^{6}$ O Brasil ocupa, atualmente, o segundo lugar em comparação ao mundo com 140.578 novos casos entre 20162018, atrás somente da Índia, e o primeiro entre as Américas6. Dentre as macrorregiões, o Nordeste é a que apresenta maior quantidade de indivíduos diagnosticados entre 20102020, seguido do Norte e Centro-Oeste, os quais, de modo geral, detêm as maiores taxas de desigualdade social no país, destacando entre esses o Maranhão, estado com a maior prevalência nesse contexto ${ }^{6}$.

$$
\text { Todavia, existem, mundial e }
$$
nacionalmente, uma série de políticas que definem estratégias e regulamentam os 
serviços de saúde no combate, diagnóstico e prevenção da hanseníase. Dentre esses, a Organização Mundial da Saúde (OMS), em 2016, implementou a "Estratégia Global para Hanseníase 2016-2020: Acelerando para um mundo livre da hanseníase", a qual prevê a redução total dos casos de hanseníase em crianças, de novos casos com deformidades visíveis e o combate à discriminação que os pacientes sofrem diariamente ${ }^{7}$. Outrossim, instituiu-se, no Brasil, o Programa Nacional de Controle da Hanseníase do Ministério da Saúde (MS), o qual preconizou a organização de forma descentralizada das medidas de saúde no combate à doença, assim como a utilização da saúde primária no rastreio e fornecimento de apoio adequado à população, buscando, por meio do monitoramento clínico através do exame neurológico simplificado, o qual verifica as sequelas provocadas pelo $\mathrm{M}$. leprae, e a vigilância laboratorial pela Pesquisa de bacilos álcool-ácido resistentes (BAAR), o controle epidemiológico da doença8. Também regulamentou-se o esquema de poliquimioterapia (PQT) do tratamento da hanseníase, o qual, dependendo da classificação em paucibacilar (1 a 5 lesões cutâneas) ou multibacilar ( $>5$ lesões cutâneas), permite a cura completa do paciente em esquemas supervisionados de 6 a 9 meses, ou de 12 a 15 meses respectivamente ${ }^{1}$.

Portanto, percebe-se que, ao avaliar o atual contexto epidemiológico da hanseníase e socioeconômico do país, um dos principais motivos para a manutenção do estado endêmico é a ineficiência do poder público na distribuição dos recursos necessários às macro e microrregiões que mais demandam a atenção estatal. Além disso, a elevada desigualdade econômica, aliada ao baixo nível escolar e acesso à informação constituem importantes fatores de agravamento e reprodução do ciclo de debilitação física, incapacidade laboral e manutenção da pobreza nos grupos populacionais mais atingidos pela hanseníase. Por fim, fica evidente que produções acadêmicas que gerem ferramentas de melhor avaliação socioespacial e auxiliem na gestão desses recursos são imprescindíveis na erradicação da hanseníase e no fornecimento de apoio e cuidados paliativos aos pacientes mais afetados. Diante do exposto o objetivo deste foi analisar o perfil clínico-epidemiológico dos casos diagnosticados com Hanseníase no Sistema de Informações de Agravos de Notificação (SINAN)

\section{MÉTODOS}

A presente pesquisa é caracterizada como um estudo observacional e retrospectivo, cujo intuito é identificar os achados clínicoepidemiológicos dos pacientes diagnosticados com hanseníase, no período de janeiro de 2011 a dezembro de 2020, os quais estavam descritos no Departamento de Informática do SUS (DATASUS), no qual foi utilizado o aplicativo TABNET que permitiu a coleta de dados cadastrados pelo Sistema de Informação de Agravos de Notificação (SINAN).

A coleta de dados ocorreu a partir do Departamento de Informática do SUS, por 
meio do Sistema de Informações de Agravos de Notificação (SINAN) (http://datasus.saude. gov.br/), na busca pelos casos de hanseníase. Os dados coletados incluíram fatores de perfil epidemiológico como: região, ano do diagnóstico, sexo, cor/raça, escolaridade, idade; e o perfil clínico: número de bacilos, classificação quanto ao tipo de hanseníase, quantidade de lesões e evolução do quadro.

Os dados foram analisados de forma descritiva e analítica. Foram verificados os dados de região, ano do diagnóstico, sexo, cor/ raça, escolaridade, idade, número de bacilos, classificação quanto ao tipo de hanseníase, quantidade de lesões e evolução do quadro. A partir da coleta de dados, eles foram armazenados em tabelas no Microsoft Excel 2019 e analisados, por meio do programa Bioestat $5.3^{\circledR}$, a partir de média, moda e variação para descrever os fatores do perfil.

Com isso, os dados obtidos foram convertidos em gráficos e tabelas utilizando os aplicativos Microsoft Excel 2019 e Microsoft Word 2019.

Foram incluídos no estudo todos os casos notificados e registrados pelo Sistema de Informações de Agravos de Notificação (SINAN) do Ministério da Saúde que foram diagnosticados com Hanseníase no período de janeiro de 2011 a dezembro de 2020.

\section{RESULTADOS}

A partir da análise de dados, foram encontrados 867.687 casos de pacientes diagnosticados com Hanseníase no período de 2011-2020, com média de 86.769 casos/ ano. No ano de 2020 houve uma redução de aproximadamente $67 \%$ em relação ao ano anterior, correspondendo ao ano de menor incidência. Ademais, foi observada uma redução dos casos em relação aos anos (GRÁFICO 1).

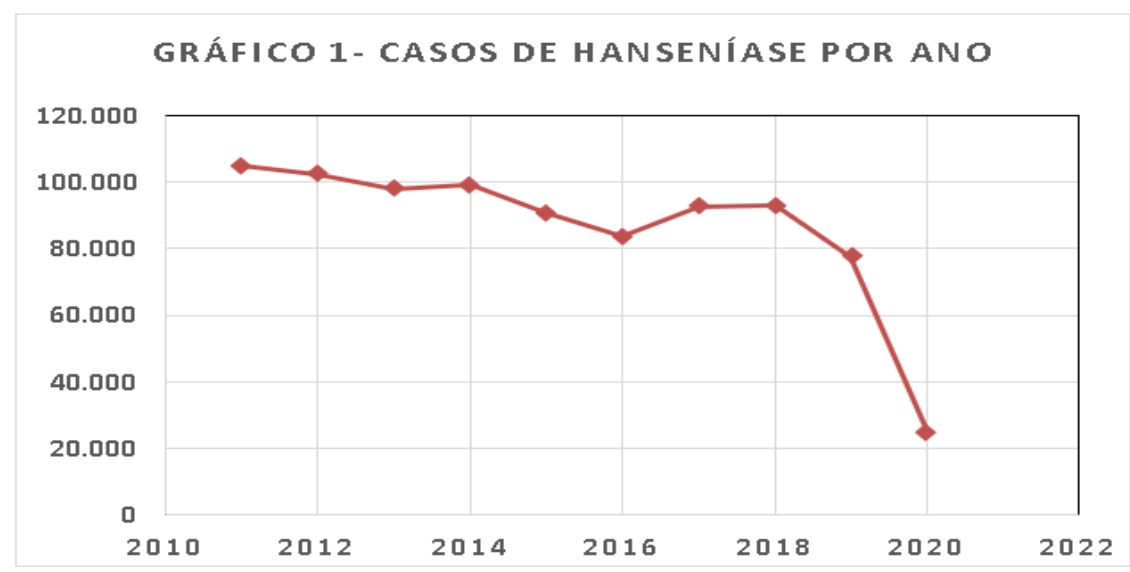

Fonte: Elaborado pelos autores 
Em relação à distribuição geográfica, foi identificado que as regiões Nordeste e Norte são as mais prevalentes, correspondendo juntas a $63 \%$ dos casos de hanseníase no país. A Região Nordeste apresentou em todos os anos acima da média de casos por ano e por região $(\mathrm{Me}=17.354)$, sendo a única região a apresentar tais dados, enquanto a região Sul foi a de menor índice. Os Estados do Nordeste com maior percentual foram Maranhão e Pernambuco, correspondendo a 29,4\% e 21,2\% respectivamente.
Ao analisar a variável sexo, foi possível identificar predominância no sexo masculino, o qual obteve maior incidência de casos em todos os anos pesquisados, mantendo uma média de 48,349 casos/ ano (GRAFICO 2). Quanto à cor/raça, foi observado que houve predomínio da parda ( $n=504.043)$, a qual correspondeu a $58 \%$ dos casos, tendo predomínio em todos os anos analisados, seguida da população branca $(24,1 \%)$. A população indígena teve o menor percentual com $0,6 \%$.

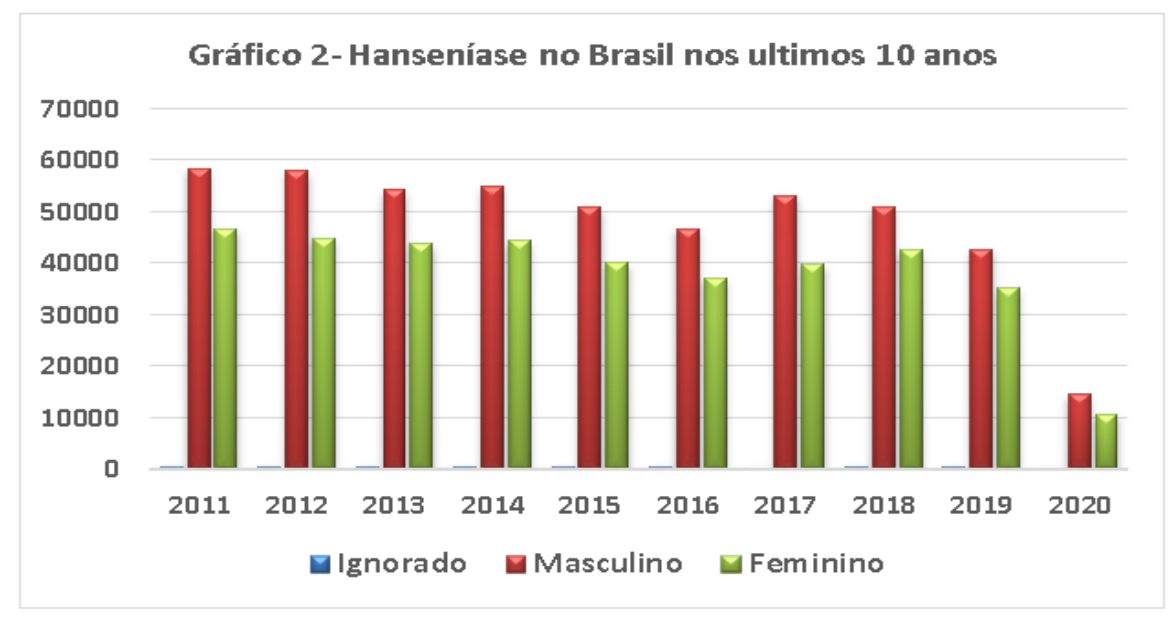

Fonte: Flahorado nelos autores

Quanto ao fator escolaridade, foi perceptível maior incidência nos níveis de base. Mais de $60 \%$ dos casos são correspondentes do analfabetismo ao ensino fundamental completo, em que há destaque a pacientes com $1^{\mathrm{a}}$ a $4^{\mathrm{a}}$ série do ensino fundamental $(n=190.373 / 21,9 \%)$. A menor incidência foi em pessoas com educação superior incompleta com $1,2 \%$.
Em relação à idade, foi predominante na faixa etária de 30 a 39 anos e de 40 a 49 anos, correspondendo respectivamente 17,9\% e 17,9\%. Também é possível observar um aumento linear de casos conforme a idade até os 49 anos, seguindo para um declínio do número de casos com uma variação negativa ao comparar com o ano anterior (TABELA 1). 
Tabela 1-Casos de Hanseníase distribuídos por faixa etária

\begin{tabular}{cccc}
\hline Idade & $\mathbf{N}$ & $\%$ & Variação \\
\hline 0 a 4 anos & 2.299 & $0,3 \%$ & - \\
\hline 5 a 9 anos & 21.198 & $2,4 \%$ & $89 \%$ \\
\hline 10 a 14 anos & 45.994 & $5,3 \%$ & $54 \%$ \\
\hline 15 a 19 anos & 46.980 & $5,4 \%$ & $2 \%$ \\
\hline 20 a 29 anos & 10.6459 & $12,3 \%$ & $56 \%$ \\
\hline 30 a 39 anos & 155.561 & $17,9 \%$ & $32 \%$ \\
\hline 40 a 49 anos & 154.916 & $17,9 \%$ & $0 \%$ \\
\hline 50 a 59 anos & 148.180 & $17,1 \%$ & $-5 \%$ \\
\hline 60 a 69 anos & 106.523 & $12,3 \%$ & $-39 \%$ \\
\hline 70 a 79 anos & 58.037 & $6,7 \%$ & $-84 \%$ \\
\hline 80 anos e mais & 21.532 & $2,5 \%$ & $-170 \%$ \\
\hline
\end{tabular}

Ademais, ao analisar os fatores clínicos, observou-se que quanto ao número de bacilos, houve prevalência dos casos de multibacilares correspondendo a $73,82 \%$ e média de 64.053 casos/ano. Contudo, no ano de 2020 a diferença percentual entre os casos tornou-se menor, com tendência a diminuir.
Em relação à classificação do tipo de Hanseníase, a Dimorfa apresentou maior incidência, seguida da Virchowiana (18,9\%). A Tuberculóide e a Indeterminada, as quais tiveram apenas uma diferença de 62 casos, sem significância percentual $(13,6 \%$ e 13,6\% para cada um), tiveram a menor incidência (GRÁFICO 3).

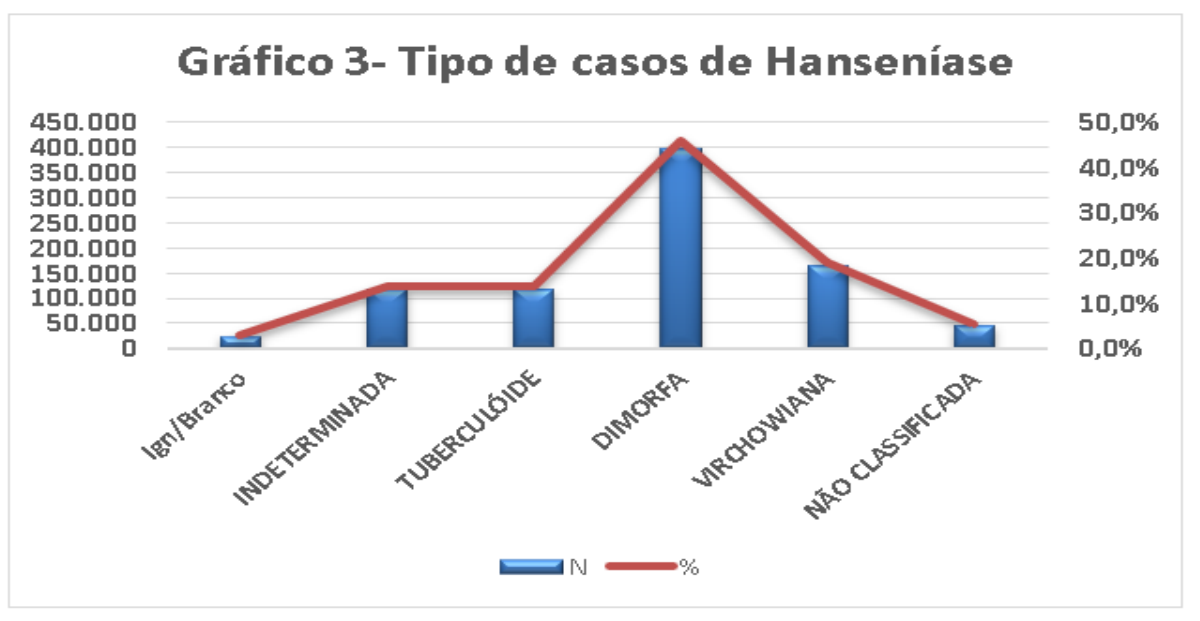

Fonte: Elaborado pelos autores. 
Quanto à quantidade de lesões apresentadas, pode-se observar uma prevalência de casos com mais de 5 lesões presentes, com média de 32.528 casos/ano com essa característica presente. Pacientes de lesão única foram a minoria, correspondendo apenas $18,2 \%$ dos casos.

Em relação à evolução dos pacientes, $80 \% \quad(n=694.637)$ evoluíram para cura, enquanto que 3,99\% foram abandonados e 1,61\% evoluíram para óbito. Essa variável apresentou um percentual considerável de evolução não preenchida, correspondendo nos 10 anos de pesquisa a 8,34\%; ademais, foi perceptível maior falta de preenchimento em 2020, o qual foi o desfecho de $83,88 \%$ dos pacientes do referido ano.

\section{DISCUSSÂO}

A notificação dos casos de Hanseníase ao Sistema de Informação de Agravos de Notificação (SINAN) é de suma importância para a vigilância epidemiológica e, portanto, para o enfrentamento da doença no País'. O enfrentamento da Hanseníase é uma meta global estabelecida, a qual já foi alcançada por alguns países no ano de 2005, no Brasil a meta não foi alcançada, sendo ampliada para 2010 e posteriormente para 2020 ${ }^{10}$. Atualmente se encontra a Estratégia Nacional para Enfrentamento da Hanseníase 2019 2022, a qual objetiva reduzir a Hanseníase no País até 2022, alicerçado na Estratégia Global para a Hanseníase 2016-2020 da Organização Mundial da Saúde 6 .

Diante dos esforços para o enfrentamento da Hanseníase no País observase redução gradual da incidência da doença no País, no entanto o Brasil manteve-se como área de alta endemicidade de acordo com o parâmetro do Ministério da Saúde para este indicador 6,11,12. Isso demonstra a gravidade da situação, tendo em vista a capacidade de a doença gerar incapacidades físicas ${ }^{13}$. Ademais, destaca-se que uma redução na incidência não deve ser o único parâmetro analisado no progresso de eliminação da doença devido à influência de outros fatores, como falha operacional, falha de notificação, culminando em falhas na notificação da doença ${ }^{10,11}$

A redução de aproximadamente $67 \%$ em relação ao ano anterior de casos no ano de 2020 coincide com a pandemia de COVID-19. Conforme dados publicados por Santos et al. no estado do Tocantins a incidência de Hanseníase até o mês de março se equiparava aos anos anteriores, porém em março de 2020 houve uma brusca redução de casos coincidindo com o aumento do número de casos de COVID-19 ${ }^{14}$. O primeiro caso de COVID-19 no Brasil foi registrado em fevereiro de 2020 em São Paulo e diante do cenário de pandemia medidas de proteção foram adotadas para controle da curva epidêmica, causando impacto em diversas áreas, especialmente na saúde, repercutindo no acompanhamento de doenças crônicas. Além disso, a possibilidade 
do diagnóstico e tratamento tardio ou incompleto decorrentes da redução do fluxo de atendimentos implica em mais complicações decorrentes da hanseníase ${ }^{15}$.

Entre as regiões brasileiras são encontradas disparidades na distribuição da Hanseníase, com concentração de casos nas regiões Norte e Nordeste e menor registro de casos na região Sul. Essa diferença reflete a desigualdade econômica entre essas regiões com baixa prevalência onde há maior desenvolvimento econômico e alta prevalência onde há menor desenvolvimento ${ }^{10}$. O estado do Maranhão, localizado no Nordeste, com maior percentual de casos, apresenta 98 dos 217 municípios classificados como hiperendêmicos ${ }^{16}$.

O maior acometimento da hanseníase ocorreu na faixa etária de 30 a 49 anos, com uma população economicamente ativa. Ainda, destaca-se a ocorrência de casos em menores de 15 anos que demonstra transmissão ativa e recente de infecção" ${ }^{9}$, sendo um indicador importante na detecção de fontes ativas e permitindo avaliar o alcance da eliminação da Hanseníase, portanto avaliando a situação da endemia ${ }^{17}$. Os casos de hanseníase em menores de 15 anos estão decrescendo em todas as regiões, com flutuações nas regiões Norte, Nordeste e Centro-Oeste ${ }^{6}$ e a persistência dos casos nessa faixa etária pode evidenciar casos multibacilares não diagnosticados e tratados ${ }^{11}$.

Mais de 60\% dos casos analisados durante o período de 2011 - 2020 ocorreram em indivíduos com até o ensino fundamental completo, indicando a maior incidência de Hanseníase em indivíduos com menor escolaridade. Nesse sentido, a escolaridade é um fator socioeconômico importante na incidência de Hanseníase e a baixa escolaridade é uma condição de vulnerabilidade e de negligência, com piora da qualidade de vida, também baixa adesão ao tratamento e presença de incapacidades físicas ${ }^{18,19}$. A baixa escolaridade corrobora com a falta de compreensão sobre a doença, duração do tratamento, reconhecimento de sinais e sintomas. Ela também se relaciona com menos recursos e dificuldade de acesso à saúde ${ }^{18}$.

Ao se avaliar a incidência de casos entre os sexos encontra-se maior predominância no sexo masculino. Em geral, os homens são mais suscetíveis a adoecer, especialmente de doenças infecciosas, devido o pouco cuidado com a saúde, menor preocupação com a estética e com o corpo em relação às mulheres ${ }^{20}$. Isso demonstra um fator cultural do País e também a maior exposição masculina frente às atividades laborais e salienta ainda os homens como vulneráveis nesse cenário da doença, principalmente no acesso a serviços de saúde direcionados para os homens ${ }^{19,21}$. Destacase, ainda, que o processo de adoecimento em mulheres encontra-se com os estigmas gerados pela doença, bem como a pressão estética corporal ${ }^{16}$.

A classificação operacional da Organização Mundial da Saúde classifica 
a hanseníase em paucibacilar - pacientes que apresentam até 5 lesões - e multibacilar - pacientes que apresentam mais de 5 lesões, permitindo a orientação do esquema da poliquimioterapia. As formas clínicas paucibacilares são a hanseníase indeterminada e tuberculóide e as formas multibacilares são a hanseníase dimorfa e virchowiana22. A prevalência de casos multibacilares evidencia formas transmissíveis em circulação, tendo em vista a transmissão de a doença ocorrer por gotículas de indivíduos bacilíferos, assim como um possível atraso diagnóstico, pois é a forma mais grave do agravo ${ }^{11,23}$.

Diante do exposto percebe-se a hanseníase como um grande problema na saúde pública do Brasil. Nesse sentido, o conhecimento do contexto epidemiológico é primordial para a elaboração de políticas públicas nas macro e microrregiões, a fim de interromper o ciclo de transmissão da doença e impedir os agravos decorrentes dela, como a incapacidade física, laboral e a manutenção da pobreza. Por fim, destaca-se que a utilização de dados secundários limita a pesquisa em virtude de variáveis de preenchimento dos dados para registro.

\section{CONCLUSÃO}

Os resultados deste estudo apontam para a redução dos registros da endemia de hanseníase no país, a qual pode estar relacionada tanto com o aumento da cobertura dos serviços de saúde especializados, por meio de programas específicos, com objetivos bem delimitados de redução de casos até 2022, quanto com a diminuição de acompanhamentos e diagnósticos, por conta da pandemia do COVID-19.

Para além desta redução de casos, a persistência das Regiões Norte e Nordeste com os maiores índices, em detrimento de regiões mais desenvolvidas, como a Sul, além de maior proporção entre pessoas de baixa escolaridade e pardos, evidencia a necessidade de se levar em consideração a pobreza e desigualdades sociais existentes nas diferentes localidades.

Assim, sugere-se a integração das ações dos serviços de saúde com ampliação de programas sociais, definindo áreas e grupos prioritários para intervenção, segundo os diversos aspectos epidemiológicos atuais e regionais, acreditando-se que a melhoria das condições de vida da população é ainda fundamental para a interrupção da transmissão continuada da hanseníase e, consequentemente, para o controle da endemia.

Espera-se com este estudo contribuir para comunidade científica, por meio de reflexões no processo de formação em saúde e representantes sociais, na discussão sobre a importância de investimentos nas ações de saúde, políticas inclusivas e planejamento adequado, com vistas a minimizar as arbitrariedades existentes em território nacional e consequentemente controlar a endemia. 


\section{REFERENCIAS}

1- BRASIL. Ministério da Saúde. Secretaria de Vigilância em Saúde. Departamento de Vigilância das Doenças Transmissíveis. Guia prático sobre a hanseníase. Brasília, 2017.

2- NIITSUMA, E. N. A. et al. Fatores associados ao adoecimento por hanseníase em contatos: revisão sistemática e metanálise. Revista Brasileira de Epidemiologia (online). v. 24, e210039. p.1-16, janeiro, 2021.

3- SOUZA, C. D.; MAGALHÃES, M. A.; LUNA, C. F.; Hanseníase e carência social: definição de áreas prioritárias em estado endêmico do Nordeste brasileiro. Rev Bras Epidemiol (Impr.) v.23: e200007, p.1-14, 2020. 4- SANTANA, E.M.F.; et al. Factors associated with the development of physical disabilities in Hansen's disease. Rev Inst Med Trop Sao Paulo, v.60, e27, p. 1-7, maio, 2018.

5- BARBOSA, C.C; et al. Spatial analysis of reported new cases and local risk of leprosy in hyper-endemic situation in Northeastern Brazil. Trop Med Int Health, v.23, n.7, p.748-757, julho, 2018.

6- BRASIL. Ministério da Saúde. Secretaria de Vigilância em Saúde. Boletim Epidemiológico Hanseníase 2020. Brasília,2020.

7- World Health Organization. Estratégia global para hanseníase 2016-2020: aceleração rumo a um mundo sem hanseníase [Internet]. Disponível<https: / / apps.who.int/ iris/bitstream/ handle/10665/250119/9789290225607Por.pdf;sequence=13>. Acesso em: 15 de ago. de 2021 8- MARQUES, N. P., et al. Impact of the coronavirus disease 2019 on the diagnoses of Hansen's disease in Brazil . Revista da Sociedade Brasileira de Medicina Tropical (Internet). 2021; 54(1).

9- BRASIL. Ministério da Saúde (MS). Secretaria de Vigilância em Saúde. Departamento de Vigilância em Doenças Transmissíveis. Plano integrado de ações estratégicas de eliminação da hanseníase, filariose, esquistossomose e oncocercose como problema de saúde pública, tracoma como causa de cegueira e controle das geohelmintíases: plano de ação 2011-2015. Brasília: Ministério da Saúde, 2012.

10- RIBEIRO, M.D.; SILVA, J.C.; OLIVEIRA, S.; Estudo epidemiológico da hanseníase no Brasil: reflexão sobre as metas de eliminação. Revista Panamericana de Salud Pública, V.42, p. 1-7, 2018.

11- BASSO, M.E.M.; ANDRADE, R.F.; SILVA, R.L.F. Trend of epidemiological indicators of leprosy in an endemic state of the Amazon region. Revista Gaúcha de Enfermagem, v.42, p. 1-11, setembro,2021

12- BRASIL. Ministério da Saúde (MS). Secretaria de Vigilância em Saúde. Departamento de Vigilância das Doenças Transmissíveis. Diretrizes para vigilância, atenção e eliminação da Hanseníase como problema de saúde pública: manual técnico-operacional [recurso eletrônico]. Brasília: Ministério da Saúde, 2016.

13- BRASIL. Ministério da Saúde (MS). Secretaria de Vigilância em Saúde. Departamento de Vigilância das Doenças Transmissíveis. Diretrizes para vigilância, atenção e eliminação da Hanseníase como problema de saúde pública: manual técnico-operacional [recurso eletrônico]. Brasília: Ministério da Saúde, 2016.

14- SANTOS, G.C.A et al. COVID-19 and the incidence of neglected tropical diseases: reflections from pandemic times. Abcs Health Sciences,, v. 46, p. 1-3, março, 2021. 15- SILVA, J.M.S et al. Atenção às pessoas com hanseníase frente à pandemia da covid-19: uma revisão narrativa. Revista Eletrônica Acervo Saúde, v. 13, n. 2, p. 1-8, fevereiro. 2021.

16- GONÇALVES, J.R.S. et al. Perfil epidemiológico e clínico da Hanseníase no estado do Maranhão no período de 2008 a 2017. Brazilian Journal Of Health Review, Curitiba, v.4, n.4, p.17015-17027, julho, 2021. 17- SCHNEIDER, P.B; FREITAS, B.H.B.M.; Tendência da hanseníase em menores de 15 anos no Brasil, 20012016. Cadernos de Saúde Pública, v. 34, n. 3, p.16784464, março, 2018.

18- LEANO, H.A.M. et al. Socioeconomic factors related to leprosy: an integrative literature review. Revista Brasileira de Enfermagem, v.72, n.5, p.14051415, outubro, 2019.

19-SOARES, G.M.M.M.; etal. Fatores sociodemográficos e clínicos de casos de hanseníase associados ao desempenho da avaliação de seus contatos no Ceará, 2008-2019. Epidemiologia e Serviços de Saúde, v.30, n.3, p.1-12, agosto, 2021.

20 -ALVES, J.M; RODRIGUES, R.P; CARVALHO, M.C.S. Perfil epidemiológico e espacial dos casos novos de hanseníase notificados em Feira de Santana no período de 2005- 2015. Revista Pesquisa em Fisioterapia, v.11, n.2, p. 334-341, maio, 2021.

21- SOUZA, E.A.; de et al. Leprosy and gender in Brazil: trends in an endemic area of the northeast region, 2001-2014. Revista de Saúde Pública, v.52, n.20, p.1-12, fevereiro, 2018.

22- VELÔSO, D.S.; et al. Perfil Clínico Epidemiológico da Hanseníase: uma revisão integrativa. Revista Eletrônica Acervo Saúde, v. 10, n. 1, p. 1429-1437, 2018. 23- BRASIL. Ministério da Saúde (MS). Secretaria de Vigilância em Saúde. Departamento de Vigilância das Doenças Transmissíveis. Exercício de Monitoramento da Eliminação da hanseníase no Brasil - LEM-2012. Brasília: Ministério da Saúde, 2015.

OBSERVAÇÃO: Os autores declaram não existir conflitos de interesse de qualquer natureza. 